\title{
Resonant elastic X-ray scattering in chemistry and materials science
}

\author{
J.R. Helliwell ${ }^{1}$, M. Helliwell ${ }^{1}$, a, V. Kaucic ${ }^{2}$, and N.Z. Logar ${ }^{2}$ \\ 1 School of Chemistry, University of Manchester, M13 9PL, UK \\ 2 The National Institute of Chemistry, Hajdrihova 19, SI-1000 Ljubljana, Slovenia
}

Received 15 November 2011 / Received in final form 23 March 2012

Published online 15 June 2012

\begin{abstract}
The applications of anomalous scattering for locating metal atoms and discriminating between different elements has increased when optimised with synchrotron X-radiation.The on-resonance effect enhances the targeted elemental signal and allows small occupancies to be determined, including in situations of a mixed metal population at a single atomic site. Thus the applications of resonant elastic $\mathrm{X}$-ray scattering in biological, inorganic and materials chemistry is being widely applied to single crystals, which is our emphasis, but also powders, fibres, solutions, amorphous and thin film states of matter. Recent developments have included the use of high photon energies (upto $100 \mathrm{keV}$ ) as well as softer $\mathrm{X}$-rays $(2 \mathrm{keV}$ ). The various instrument and technical capabilities have improved in the last 15 years. This ease of measurement of the resonant scattering signals along with absorption edge shifts indicates an expansion to the measurement of multiple data sets, to allow monitoring of redox changes. Whilst crystal structure determination in biological crystallography has been revolutionised by the MAD method, it is not a requirement for chemical or materials crystallography, as other phasing techniques are routine. Synchrotron source upgrades will allow nano-sized X-ray beams to be more widely available. The new X-ray lasers suggest new capabilities too.
\end{abstract}

\section{Introduction}

Anomalous scattering results for locating metal atoms and discriminating between different elements has increased and found wide application in chemistry and materials science from the optimisation via X-ray wavelength tuning with synchrotron radiation. The on-resonance enhances the targeted elemental signal and allows even small occupancies to be determined, including in mixed metal situations. The polarisation state of the synchrotron X-ray beam also allows dichroism effects to be explored, although the range of applications is less extensively developed as yet. The applications of resonant elastic X-ray scattering include the crystallography of microporous materials, superconductors and magnetic materials as well as in the biological chemistry of metalloproteins.

\footnotetext{
a e-mail: john.helliwell@manchester.ac.uk
} 
With inorganic and materials chemistry, the number of wavelengths used is increasing and the ease of measuring diffraction data to usual chemical crystallography standards has improved, even with longer wavelengths. The various instrumental and technical capabilities have improved in the last 15 years and case studies are described in Sects. 4 and 5; a recent example involved use of eleven X-ray wavelengths, each with diffraction data recorded to a usual, high, standard (at $0.84 \AA$ resolution) to study a zinc substituted gallophosphate. This ease of measurement of the resonant scattering signals along with absorption edge shifts indicates an expansion to multi-data sets measurement to allow monitoring of redox changes, as a function for example of $\mathrm{pH}$ or temperature. Whilst crystal structure determination in biological crystallography has been revolutionised by the synchrotron MAD (Multiple-wavelength Anomalous Dispersion) method [see for example ref 1 for an early example] it is not a requirement for chemical or materials crystallography, as other phasing techniques are routine i.e. such as direct methods using single wavelength, non-resonant condition diffraction data. Further applications to powders, solutions, thin films and amorphous materials indicate a buoyant contribution of Resonant Elastic X-ray Scattering (REXS) to chemistry and materials characterisation of atomic sites.

Binuclear cases of lectin proteins and enzymes exist, with metals such as manganese and calcium, or copper with zinc, and the metal sites are difficult to distinguish without resonant scattering. A prominent recent biological example [2], where X-ray radiation damage was a real challenge, solved by a special data collection scheme, was the oxygen evolving complex $\mathrm{Mn}_{4} \mathrm{Ca}$, with nearby chloride ions, and the crystallography analyses involved X-ray data collected near to the manganese $\mathrm{K}$ edge. Recent technical developments in biological chemistry have included the use of high photon energies (upto $56 \mathrm{keV}[3]$ ), although applications to powders have reached upto even higher photon energies $(\sim 90 \mathrm{keV}[4])$, as well as softer X-rays $(2 \mathrm{keV})$ [reviewed in ref [5]] thus widening the elements range of application.

A selection of the relevant literature overviews include the following references; the previous two conferences [6,7], a review of SR X-ray and neutron methods [8], applications of resonance diffraction, including DAFS (Diffraction Anomalous Fine Structure) [9], determination of absolute molecular structure ie 'the hand' [10], development of instrumentation and methods for MAD at various SR facilities [11] and an extensive recent survey of applications to small molecules and proteins, including an overview of complementary techniques [12].

In contrast to the use of MAD techniques in protein crystallography, since the majority of small molecule samples using MAD are centrosymmetric, experiments are generally carried out to induce changes in $f^{\prime}$, rather than $f^{\prime \prime}$. The experiments still of course involve the collection of one or more data sets close to the absorption edge of the element(s) in question, together with a reference data set, remote from the absorption edge(s). The differences in the $f^{\prime}$ value between the various wavelengths then allow the sites and / or valence states of the target elements to be determined; case studies are highlighted later in this article. In recent years a number of additional diffraction and spectroscopic techniques have been developed which rely on the fact that the precise shape of the absorption edge is sensitive to the environment of the atom in terms of both the geometry of the coordination and the types of atoms in the first and second coordination shells. These techniques are able to probe the coordination geometry and valence states at particular sites within the structure (using X-ray Absorption Spectroscopy (XAS) and DAFS [9,12]). For conventional $\mathrm{X}$-ray sources i.e. at a fixed wavelength, use is made of non-wavelength optimised $f^{\prime \prime}$ of specific atoms, to determine the absolute configuration of chiral small molecules; these cases are very numerous, but as they do not involve on-resonance elastic X-ray scattering (REXS) techniques they are outside the scope of this article (for further details see $[10,12])$. Enhancement of the magnetic X-ray scattering effect also exists 
via REXS but is considered specifically in the dedicated papers [13,14]. Characterization of nano-structures in semiconductors are described in the companion paper in these proceedings [15].

\section{Some basics}

The overall X-ray scattering effect of an atom is described by the following atomic scattering factor equation and includes wavelength and scattering angle dependencies:-

$$
\mathrm{f}(\lambda, \theta)=\mathrm{f}_{0}(\theta)+\mathrm{f}^{\prime}(\lambda)+\text { if }^{\prime \prime}(\lambda)
$$

$f^{\prime}$ and $f^{\prime \prime}$ are usually called the anomalous scattering corrections to the normal scattering factor of an atom $\mathrm{f}_{0}$. Of course real scattering involves all the terms in Eq. (1) and are not anomalous at all. Sometimes, in a genuine attempt to adopt a more correct nomenclature [12], the 'anomalous scattering' word is replaced by 'resonant scattering'. This terminology has been used even when 'off-resonance' [16]. The offresonance case is particularly relevant to laboratory X-ray sources using eg Mo or $\mathrm{Cu} \mathrm{K}$ alpha radiation. Basically the wordings in common use are none of them ideal. Experiments done at the X-ray absorption edge are clearly on-resonance and it is these latter cases that are the subject of this article, as already mentioned above.

The dependence on $\theta$ occurs mainly through $\mathrm{f}_{0}$, again as indicated in Eq. (1), which decreases with increased angle. Since $f^{\prime}$ and $f^{\prime \prime}$ arise mainly from inner shell electron effects, i.e. over small distances from an atom's nucleus, their fall-off with $\theta$ is much less marked than for $\mathrm{f}_{0}$. Each of the terms is weakened by atomic mobility and thereby accentuates the decrease with $\theta$ in the X-ray scattering involving all three terms in Eq. (1), for a given atom.

There are absorption edge shifts for a particular metal atom oxidation state; e.g. manganese with its various oxidation states makes a nice chemical illustration so that the edge shifts for oxidation states $2,3,4$ and 7 are respectively approximately $4 \mathrm{eV}$, $8 \mathrm{eV}, 11 \mathrm{eV}$ and $20 \mathrm{eV}$ [Fig. 1]. These edge shifts emphasise why it is critical to locate the actual absorption edge in a sample study of interest so as to be actually 'on edge'.

There are orientational effects, known as dichroism, [18] that require Eq. 1 to be treated as a tensor [19] and for the state of polarisation of the incident beam to be properly characterised.

The range of photon energies harnessed is now very wide spanning soft X-rays such as $\sim 2 \mathrm{keV}$ to access sulphur or phosphorus $\mathrm{K}$ edges [5], and high photon energies to access high Z K-edges such as bismuth and lead [4].

\section{Instrumentation overview}

We briefly summarise a range of developments. The evolution of tunable X-ray synchrotron sources, including the advent of the undulator, have widened the scope considerably, notably to smaller samples. A wide range of X-ray wavelengths have now been accessed from high photon energies (e.g. upto $100 \mathrm{keV}$ ) to lower energies (e.g. to $2 \mathrm{keV}$ ). The diffractometer or apparatus at the beamline can be a significant limitation; for chemical crystallography, initially single counter four circle diffractometry was superseded by adapting protein crystallography area detector methods, but had limited detector angular acceptances; more recently, dedicated chemical crystallography apparatus has become available, with full back-scattering capability. As well as single crystal and powder applications, these are now extended to other states of matter, such as solutions and thin films. Improved ease of measurement 


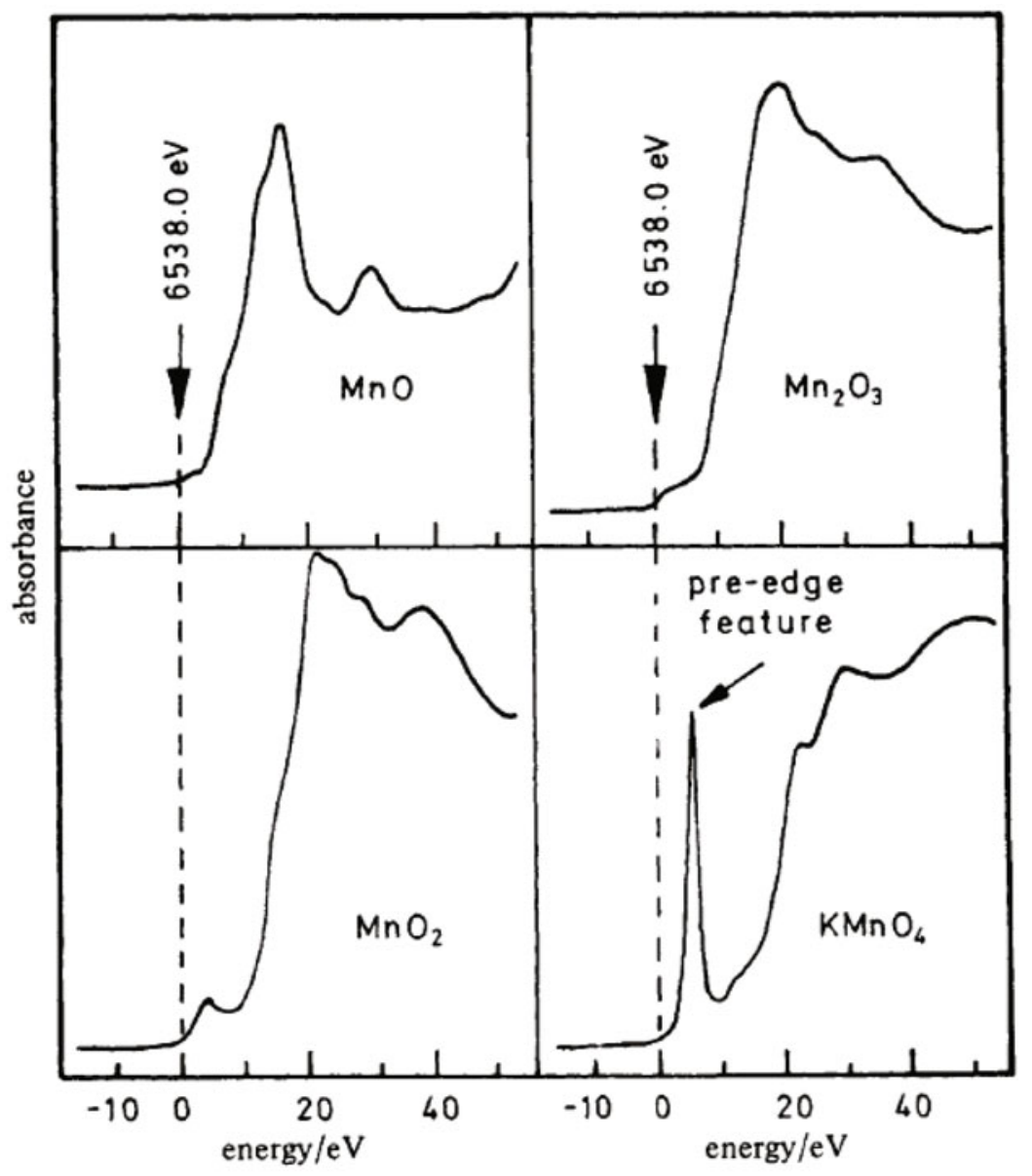

Fig. 1. XANES spectra of the reference oxides (a) $\mathrm{MnO}$ (b) $\mathrm{Mn}_{2} \mathrm{O}_{3}$ (c) $\mathrm{MnO}_{2}$ and (d) $\mathrm{KMnO}_{4}$ representing valence states 2, 3, 4 and 7 of manganese respectively and their associated edge shifts. Reproduced with permission of the authors and The Royal Society of Chemistry [17].

has allowed multiple data sets to be measured, so as to study variation of temperature to assess redox changes, in a site specific way [20]. Dichroism applications are increasingly underway, utilising the SR X-ray beam polarisation $[18,19]$. DAFS (DANES i.e. Diffraction Anomalous Near Edge Structure)) combines crystallography and X-ray spectroscopy into the same method (for a detailed description and review see $[9])$.

As part of the tutorial aspect of this article, Fig. 2 shows a state-of-the-art beamline optics configuration at Diamond Light Source (DLS) suitable for rapid wavelength tuning (via its double crystal monochromator) for REXS studies.

Its beamline specification is as follows:-

- Double monochromator for rapid wavelength tuning

- Energy range: 5 to $25 \mathrm{keV}, 0.5$ to $2.5 \AA$ 


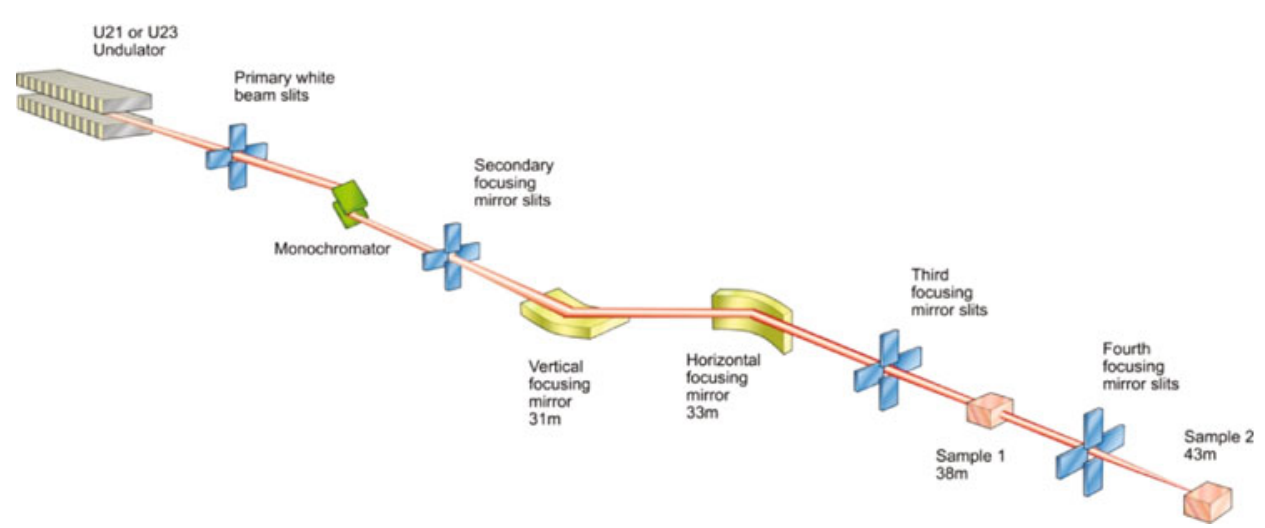

Fig. 2. The beamline scheme for the DLS I19 for chemical crystallography including REXS as an illustration of a state-of-the-art beamline. With the permission of Diamond Light Source and Professor Dave Allan, Scientist in charge.

- Energy resolution $(\Delta \mathrm{E} / \mathrm{E}):<2 \times 10^{-4}$

- Energy stability $(\Delta \mathrm{E} / \mathrm{E}):<4 \times 10^{-5}$

- 4-circle kappa goniometer with CCD detector for data collection to high resolution.

The instrumental challenges for using low photon energies include a lack of diffraction resolution ie where even with back scattering the smallest $d$ spacing measurable is obviously no better than $\lambda / 2$ and the need for a special diffractometer enclosure either of a helium atmosphere or a vacuum. The benefit however is that low atomic number elemental sites can thereby be accessed and may indeed be the natural ones present [5].

The instrumental challenges for using high photon energies include poor detector absorption efficiency, and access is restricted to just a few SR facilities (namely those with a high machine energy and/or high magnetic field insertion device). The benefit from such experiments can arise though when absorption from the sample or sample environment/container is a major barrier to the acquisition of high-quality resonant scattering data at lower-energy edges [5].

\section{Chemical case studies: The structure and function of microporous materials and technical requirements for REXS chemical crystallography}

Redox microporous catalysts are recenty the most popular, environmentally friendly, catalysts [21] e.g. for oxidations with $\mathrm{O}_{2}$ or $\mathrm{H}_{2} \mathrm{O}_{2}$ (i.e. advanced oxidation processes (AOPs) in waste-water treatment, decoloration of dyes, degradation of organic pollutants (e.g. endocrine disruptors, etc.) to $\mathrm{CO}_{2}$ and $\mathrm{H}_{2} \mathrm{O}$ ). The incorporation of transition metals into silicate, aluminosilicate, aluminophosphate and similar inorganic microporous and mesoporous frameworks generates and/or moderates their redox and acid catalytic properties. The advantages of the so-prepared single-site catalysts with discrete active metal sites that mimic enzyme function have extremely high selectivities, leading to the production of sharply defined molecular products. Transition metal ( $\mathrm{Mn}, \mathrm{Co}, \mathrm{Fe}, \mathrm{Ti}, \mathrm{V}, \mathrm{Cr}$, etc.) modified zeolites and microporous silicates catalyse a variety of selective oxidations and reductions under mild conditions, with the advantage of facile recovering and recycling, when compared to homogeneous 
liquid phase catalysts, such as sulphuric acid. The most important example is Timodified silicalite-1, which is an excellent catalyst with selective properties for epoxidation of olefins using $\mathrm{H}_{2} \mathrm{O}_{2}$. Zeolite Beta and ZSM-5 modified with Fe, Mn or Co also show good catalytic activity for hydrocarbon oxidations and reductions. Metalmodified $\mathrm{AlPO}_{4}-n$ microporous catalysts oxidise linear alkanes using molecular oxygen as reagent. MeAPO-36 $(\mathrm{Me}=\mathrm{Mn}, \mathrm{Co})$ is a bi-functional catalyst for converting cyclohexanone to $\varepsilon$-caprolactam, where mutually present $\mathrm{Me}^{2+}$ and $\mathrm{Me}^{3+}$ ions act as Brønsted and redox sites, respectively. Direct oxidation of cyclohexene with aqueous $\mathrm{H}_{2} \mathrm{O}_{2}$ to adipic acid, which is used in the production of nylon, has been performed with different Ti- or Fe-substituted silicate and aluminophophate microporous catalysts. An example of a redox catalyst is where $\mathrm{Mn}^{3+}$ replaces $\mathrm{Al}^{3+}$ (usually up to a few percent; most structures are not flexible enough to accomodate more metal) and the manganese is able to change the oxidation state from $3+$ to $2+$ and vice-versa. In summary we can say that:- Incorporation of transition metals crucially affects the activity of microporous catalysts, which are still gaining importance in different environmental and energy-saving applications from waste-water treatment to clean production of adipic acid, fine chemicals' production, etc; the incorporation of transition metals into a framework helps the stability of the site and prevents deactivation of the catalyst. An ideal microporous catalyst is well-dispersed and stable as well as having an accessible active site.

Applications of REXS techniques give neighbouring atom contrast for fully characterising a given nanoporous catalyst. The metal atom substitution may be over more than one site, often at partial occupancy. Also more than one metal atom with similar atomic numbers may occupy the same site, making them especially difficult to determine. Specific elements can be identified at different sites and the extent of their incorporation can be determined by REXS techniques.

We emphasise that the need for the determination of the site(s) of transition-metal incorporation in the structure is :- firstly, because its framework or extra-framework location of metal influences the stability of the material; secondly, so as to determine the accessibility of the preferential site of incorporation of a metal (e.g. next to the large channel or hidden in the walls); thirdly, to correlate the overall chemical catalytic properties with the metal location, which is to enable a better selection of a suitable porous structure and type of transiton metal, for optimal catalyst activity and proper design of new catalysts. The properties of microporous materials can be modified by the incorporation of transition metals either by post-synthetic ion exchange and subsequent thermal treatment (mostly for zeolites) or by direct substitution into the framework.

A recent example of the use of REXS techniques in the study of such microporous materials involves the location of isomorphously substituted zinc over eight crystallographically different gallium sites, which was determined in a single crystal study of the gallophosphate ZnULM-5, in an eleven-wavelength experiment, using data from Station 9.8, SRS Daresbury [22], Fig. 3(a).

Since zinc and gallium are neighbouring in the Periodic Table, it was not possible to distinguish the sites of zinc incorporation by single $\mathrm{X}$-ray wavelength techniques. Also because the absorption edges are relatively close together (the $\mathrm{Zn}$ and $\mathrm{Ga}$ K-edges are at 1.2834 and $1.1958 \AA$ respectively), there tends to be contributions to the anomalous dispersion effect from both metals. In order to vary the atomic scattering factor of each element in turn, the measurement of multiple data sets around the $\mathrm{K}$ edges of both $\mathrm{Ga}$ and $\mathrm{Zn}$, as well as two reference data sets away from each absorption edge, was utilized to selectively exploit dispersive differences of each metal atom type in turn, which allowed the major sites of $\mathrm{Zn}$ incorporation to be identified as the metal 1 and 3 sites, M1 and M3 (Fig. 3(b)). The preferential substitution of Zn at these sites probably arises because they are located in molecular building units which can relax to accommodate the incorporation of hetero atoms. Because the crystal was 

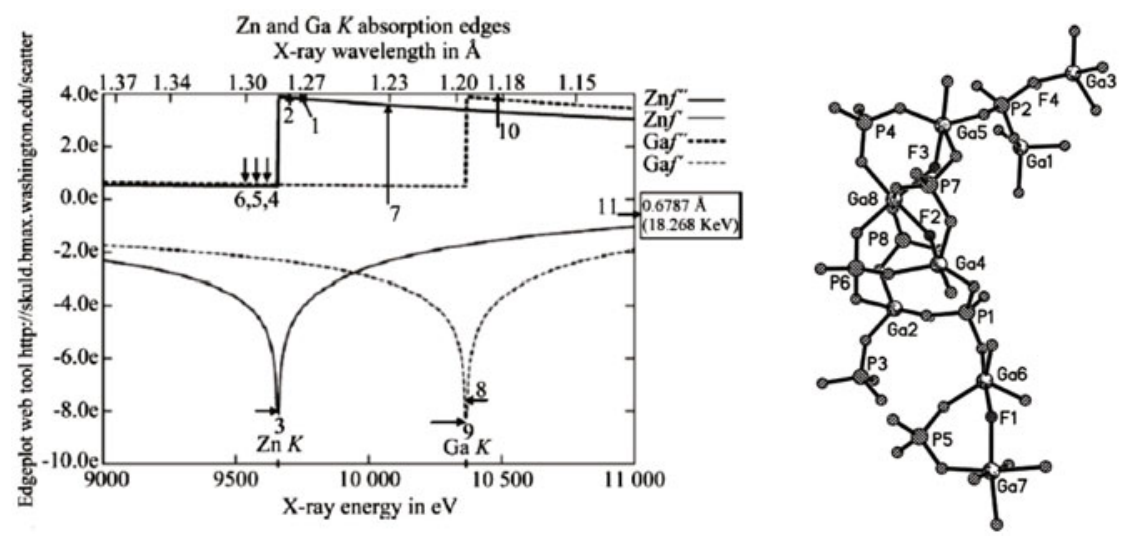

Fig. 3. (Left) the absorption edges of $\mathrm{Zn}$ and $\mathrm{Ga}$, with approximate wavelength positions of the 11 datasets collected marked. (Right) plot of a portion of the framework of ZnULM-5 showing the four-, five- and six-coordinate metal atom sites. All the $\mathrm{P}$ atoms are tetrahedral. From ref. [22] with the permission of the authors and the International Union of Crystallography.

non-centrosymmetric, with space group $\mathrm{P} 2_{1} 2_{1} 2$, it was also possible to use anomalous differences (i.e. $f^{\prime \prime}$ derived) to corroborate the results obtained from the dispersive differences. These results were obtained firstly from difference Fourier maps, calculated using a phase set from the refined crystal structure from X-ray data measured at the Zr K edge. Also, refined dispersive and anomalous occupancies, on an absolute scale, could be obtained using the program MLPHARE [23], allowing estimates for the $\mathrm{Zn}$ incorporation of approximately 22 and 18 at. $\%$ at the M1 and M3 sites to be obtained. In addition, $f^{\prime}$ and $f^{\prime \prime}$ values for $\mathrm{Ga}$ and $\mathrm{Zn}$ at each wavelength could be estimated both from MLPHARE results, and by refinement in JANA2006 [24]. Overall the fully quantitative determinations of the dispersive and anomalous coefficients for $\mathrm{Ga}$ and $\mathrm{Zn}$ at each wavelength, as well as metal atom occupancies over the eight metal atom sites, were made from use of both the CCP4's MLPHARE program as well as SHELXL [25] and JANA2006. The results by these three computational methods agreed closely, and JANA2006 allowed the ready determination of standard uncertainties on the occupancy parameters, which were for M1 and M3, 20.6 (3) and 17.2 (3) at \%, respectively, standard uncertainty values are in brackets.

Another interesting case study [20], mentioned above, is the investigation of the mixed valence complex $\mathrm{Fe}_{3} \mathrm{O}\left(\mathrm{OOCC}\left(\mathrm{CH}_{3}\right)_{3}\right)_{6}\left(\mathrm{C}_{5} \mathrm{H}_{5} \mathrm{~N}\right)_{3}$ (Fig. 4(a)), which shows a temperature dependence of the bond lengths and oxidation state, with a cross-over of the Fe(1) and Fe(3) bond lengths occurring at about $100 \mathrm{~K}$ (not shown). Similarly the REXS results measured at 18, 85, 100 and $150 \mathrm{~K}$, show a cross-over of $f^{\prime}$ values, arising from an electron transfer $\mathrm{Fe}^{2+}+\mathrm{Fe}^{3+} \rightarrow \mathrm{Fe}^{3+}+\mathrm{Fe}^{2+}$ (Fig. 4(b)); the bond lengths and $f^{\prime}$ values of the $\mathrm{Fe}(2)$ site (not shown) remain practically invariant at all temperatures, indicating a valence state of III at this site.

These and other examples prompt the question of the sensitivity to the percentage of metal substitution of the REXS approach to characterisation. An analysis of the minimum metal atom content of a single anomalous scatterer in a typical zeolite or phosphate based molecular sieve, which could be detected by anomalous scattering methods was given in Helliwell et al. [26] by determination of the dispersive differences (DD) and for chiral samples, the anomalous differences (AD) of such materials. This aspect was also addressed by Zhang et al. 2005 [4] in the context of $\mathrm{Pb}$ vs $\mathrm{Bi}$ as 
(a)

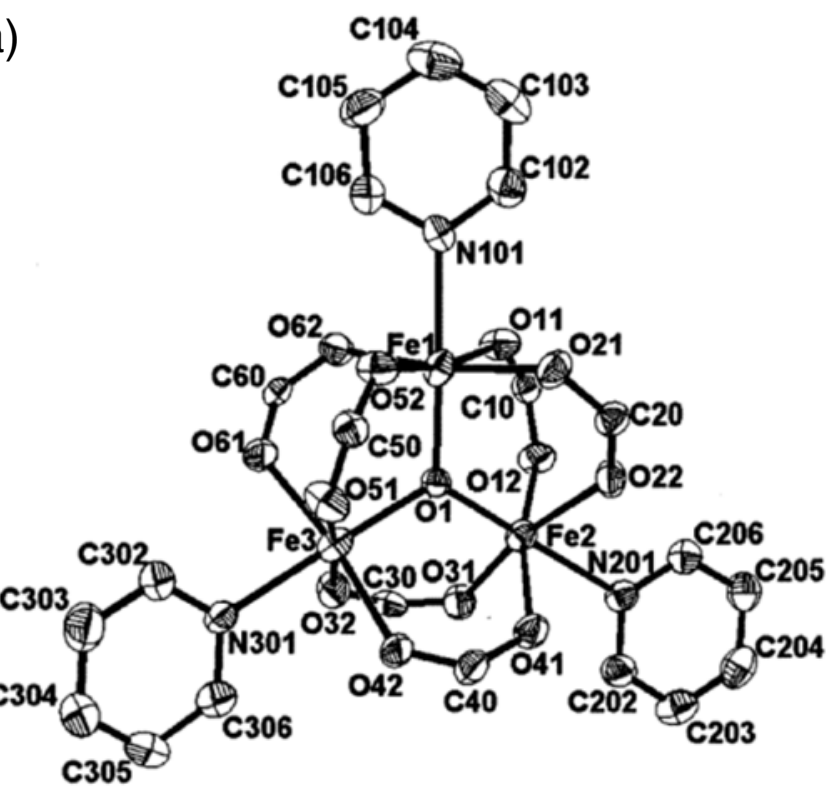

(b)
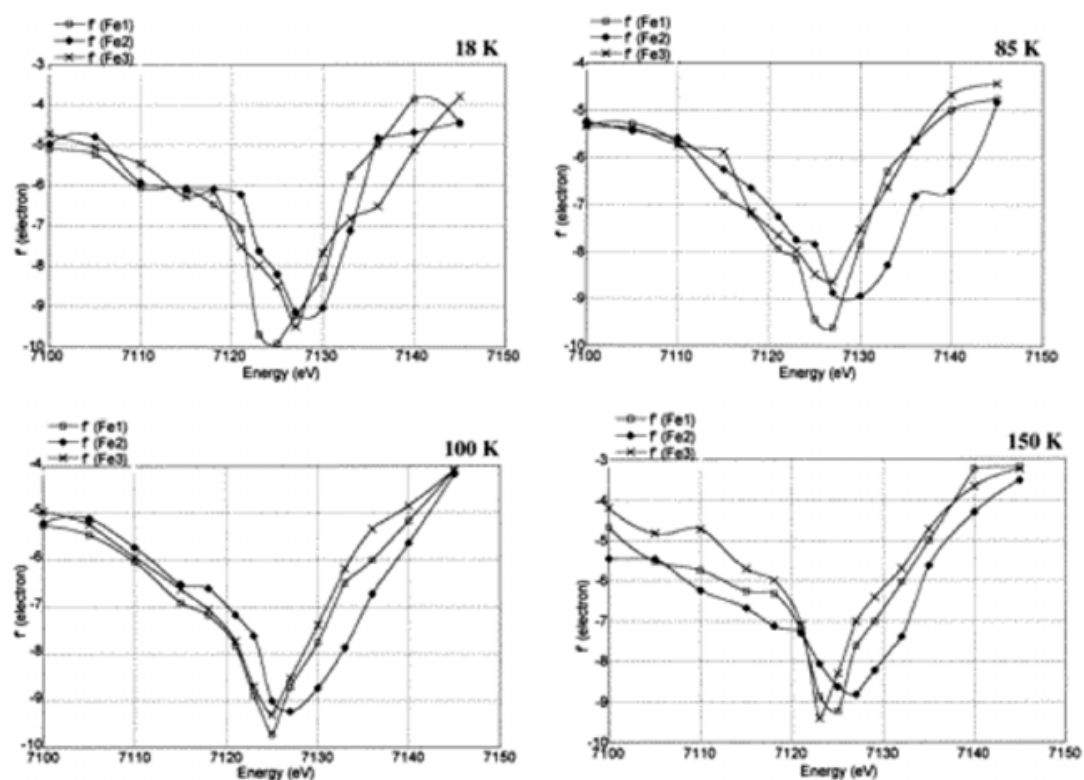

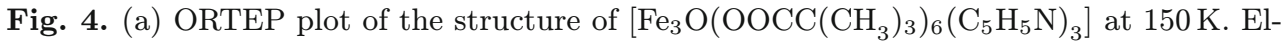
lipsoids are $50 \%$ probability surfaces. $\mathrm{H}$ atoms and butyl groups are omitted. (b) Anomalous scattering factor $f^{\prime}$ of Fe ions near the K edge as determined from refinement of diffraction data at different energies. Top row: $18 \mathrm{~K}, 85 \mathrm{~K}$. Bottom row: $100 \mathrm{~K}, 150 \mathrm{~K}$. With permission from the authors of ref 20 and Copyright 1998 American Chemical Society. 
'minimum scattering contrast' defined as:

$$
2\left[\left(f+f^{\prime}\right)_{\mathrm{Bi}}-\left(f+f^{\prime}\right)_{\mathrm{Pb}}\right] /\left[\left(f+f^{\prime}\right)_{\mathrm{Bi}}+\left(f+f^{\prime}\right)_{\mathrm{Pb}}\right] .
$$

\section{A biological chemistry case study; the $\mathrm{Mn}_{4} \mathrm{Ca}$ cluster in photosystem II (PSII)}

A notable challenge in recent years has been trying to resolve the $\mathrm{Mn}_{4} \mathrm{Ca}$ oxygen evolving complex in photosystem II (for a review see [27]). In photosystem II, there were discrepancies between crystallographic studies and inconsistencies between the $\mathrm{X}$-ray, electron paramagnetic resonance $(\mathrm{EPR})$ and Fourier transform infra-red (FTIR) spectroscopic data [28]. In particular, the model of the $\mathrm{Mn}_{4} \mathrm{Ca}$ complex from the X-ray crystallography data [29] was described as 'highly unlikely' [28]. Under the dose conditions of the X-ray measurements, the EXAFS spectrum changed significantly from one characteristic of a high-valent multinuclear oxo-bridged $\mathrm{Mn}_{4} \mathrm{Ca}$ cluster, to one that is characteristic of mononuclear hexacoordinated $\mathrm{Mn}$ (II) in solution [28]. The $\mathrm{Mn}_{4} \mathrm{Ca}$ oxygen evolving complex in Photosystem II has recently been resolved to $1.9 \AA$ [2]. To control the X-ray damage, as the authors [2] state:- "to suppress the possible radiation damage to a minimum level, we used a slide-oscillation method, resulting in the X-ray dose at each point of the crystal being lower than in previous experiments of Yano, J. et al. [28]." The Diffraction Precision Index [30,31] of the whole PSII structure was found to be $0.11 \AA$, resulting in a standard uncertainty in the bond lengths of the cluster of $0.16 \AA$. This detailed description of bond distances and uncertainties is an important step forward in its own right ie beyond the overall layout picture of the cluster, but which is also very important obviously. To describe the REXS relevant details the following diffraction data details are significant:- "For the data set taken at a wavelength of $1.75 \AA$, [i.e. for which the $f^{\prime \prime}$ value is $\sim 3.4 \mathrm{e}-$ near to the theoretical maximum for $f^{\prime \prime}$ of 3.9e- i.e. with the $\mathrm{Mn} \mathrm{K}$ absorption edge being at $1.896 \AA$ ] we collected 2,400 oscillation images, each rotated by 0.3 degrees over a range of 360 degrees. For each of the data wedges of 10 degrees, an inverse beam geometry was used to measure the Friedel pairs directly." This illustrates the wavelength tuning used (to the Mn K edge) to be close to on-resonance and also the very large quantity of diffraction data measured for such a large crystallographic unit cell.

In ref. [2] their Fig. 4(a) shows the anomalous difference Fourier map (along with the final refined structure 2 Fo-Fc map), and being contoured at 8 sigma indicates the high quality of this map and the anomalous differences derived from an $f^{\prime \prime}$ of $\sim 3.4 \mathrm{e}-$, close to the theoretical maximum of 3.9e-, as well as the use of an 'inverse beam' every 10 degrees of crystal sample rotation and a full revolution (360 degrees) of diffraction data measurements. Thus the precise location and identity of these relevant ions, have now been determined.

An early application of REXS discriminating Mn and Ca can be found in [32], the case of pea lectin; refs. [2] and [32] followed therefore the same REXS methodologies. The utility of having standard uncertainties (su's) on bond distances and angles, including around the $\mathrm{Mn}$ and $\mathrm{Ca}$ environments, in the closely related protein to pea lectin, concanavalin A, using $0.94 \AA$ resolution is described in [33]. At $1.9 \AA$ resolution, as used with PSII, the su's cannot be derived from full matrix inversion refinement techniques but instead have to use the Diffraction Precision Index approach [30,31]. This latter, although an overall structure precision, can be adopted to each atom via their individual atomic B-factors (i.e. atomic displacement parameters) relative to the average B-factor for the whole structure. 


\section{When do resonant scattering diffraction techniques fade or fail in capability? What remedy?}

There are several situations where REXS techniques in crystallography are restricted or even fail. These are as follows:-

- Cases where lower atomic number metals are of interest (e.g. titanium which has a K-edge at $2.497 \AA$ ); even back scattering measurements of the X-ray reflections will 'only' yield $1.25 \AA$ resolution); an alternative solution to obtaining site specific titanium occupancies has harnessed neutron diffraction of specifically substituted Ti isotopes in silicalite [34];

- The case of even lower atomic number e.g. aluminium (7.948 $\AA$ ) is interesting; ${ }^{27} \mathrm{Al}$ NMR studies of aluminosilicates and site-specific substitution are briefly reviewed in the context of REXS in ref. [12];

- Special apparatus for softer X-rays work, up to and including the phosphorus K edge at $5.784 \AA$, has been established at ESRF by H. Stuhrmann et al. (reviewed in [5]) and an in vacuum diffractometer, for macromolecular crystallography, is being built at Diamond Light Source by A Wagner et al., for example [35].

\section{Other REXS techniques harnessed in chemistry and materials}

In this article we have focussed on crystallography techniques. Other relevant techniques are DAFS (DANES) [36], Anomalous SAXS e.g. of soft matter [see below]; Anomalous amorphous materials diffraction $[37,38]$; X-ray magnetic scattering enhancement on-resonance [39]. All of these applications have been surveyed in detail in ref. [12]. Anomalous Grazing Incidence SAXS of nanocomposites is described in ref. [40].

A new optimized instrument for anomalous small-angle X-ray scattering has been initiated at ESRF, aiming at charged soft matter studies [41]. This is further described in the companion paper in this REXS proceedings [42]. The experimental design and setup took special care to realise single-photon detection sensitivity, a high energy resolution of the monochromator, in situ calibration of intensity and energy, and the avoidance of radiation damage to the sample, using a special sample holder. Measured intensities taken are normalized to an absolute scale online at the instrument, from which a deconvolution to resonant and non-resonant contributions is made. Thereby the anomalous scatterer signal and visualisation can then be made explicitly. The performance of this state-of-the-art instrument was demonstrated [41] by an example from soft matter materials science involving cationic surfactant micelles with bromide counter-ions. The analysis of multiple SAXS curves measured near the K-absorption edge of bromine yielded the counter-ion profile around the micelle. The results gave the radial profile of the counter-ions, showing strong condensation of the counter-ions on the micellar surface, in agreement with the inference from electrochemical methods. It is to be expected that this new instrument will serve a range of soft condensed matter, biophysical and materials chemistry SAXS research applications.

\section{Some future trends}

Dichroism effects are exploited already but we expect will expand in the number of studies undertaken in the coming years, as appropriate software becomes routinely 
available [see e.g. 19]. Chemical changes induced by temperature and/or time-resolved studies should also increase the range and scope of these REXS applications as per the case study above [20]. As the speed of measuring improves, as more instruments are available and as analysis is more routine then increasing numbers of samples can be studied per molecular system and perhaps even sample to sample variations allowed for, although unlikely to be a major concern. Protein powder diffraction harnessing dispersive differences is also under investigation [ 43 and references therein].

A major initiative at $3^{\text {rd }}$ generation SR sources, such as the ESRF Upgrade programme, emphasises nanosized X-ray beams, which when coupled with tunability will expand the REXS techniques to nano-sized materials. Such nano-size X-ray beams can help look for local variations in micro-scans across a sample (although sample thickness will remain an averaging effect).

\section{Radiation damage a limitation?}

In REXS, a wide range of photon energies are naturally harnessed. What is the radiation damage effect on each of these materials that we have described above, over this photon energy range? Might this prove to be a limitation of the applications of REXS? Is it only a biological samples concern as exemplified in the PSII studies [2,27-29] described in detail above? The ASAXS section above also suggests that in soft matter materials science studies radiation damage effects must be anticipated and minimised [41]. In our own previous research involving a nickel aluminophosphate we observed an X-ray radiation damage effect, admittedly after prolonged irradiation of 1 week at the NSLS [44] and the impact of X-rays on disulphide bridges in proteins $[45]$ is now well known.

\section{Concluding remarks and future prospects}

The field of application of anomalous scattering in structural chemistry and biology is now truly vast [12]. The evolution of capability is breathtaking and shows up the pace of modern day research. There are also complementary analytical tools available such as neutron isotopic substitution [34] and NMR [12], which have been briefly mentioned. In challenging cases, for example of particular states of dilution of metal atoms or ions, corroboration of results from using a selection of techniques together is the ideal approach.

We have explained in tutorial style the basics and range of the techniques as well as the instrumentation needed to undertake such studies. We have highlighted several case studies as well as providing a variety of references to illustrate the range and extent of REXS applications. A recent review of micro- and mesoporous materials can be found in ref. [46].

Overall, to conclude, the necessary technical advances have been made to harness $\mathrm{X}$-ray anomalous scattering these last decades, which have now brought reliable answers to a vast number of bioinorganic, inorganic and materials chemistry research challenges.

The future indicates an expansion of the field as the measurements are more rapid and there are increased numbers of SR beamlines available. Beamlines providing high and low photon energies (in a range of $\sim 90$ to $2 \mathrm{keV}$ ) indicate wide chemical element capability. The source upgrades towards providing nano-sized tunable X-ray beams, such as at ESRF, opens new possibilities of applying REXS techniques increasingly for nano-science. Also the XFELs for REXS applications in structural chemistry and biology, even perhaps with single molecules for diffraction studies, are an interesting 
prospect if the wavelength setting can be controlled, and the inner shell photoelectron ejection [47] proves not to be limiting.

$\mathrm{J}$ R Helliwell thanks all his past and current collaborators over the years in these fields. Numerous funding agency and SR beamtime awards have been received including from the SERC, the Swedish Research Council, the BBSRC, EPSRC and CCLRC UK government research agencies, and the SRS Daresbury, NSLS, ELETTRA and ESRF, and to whom JRH is especially grateful. The anomalous scattering work has evolved over a period of 30 years and so JRH is grateful to the Universities of Oxford, Keele, York and Manchester and to the Daresbury Laboratory for providing stimulating environments in which to study and to do experiments. V. Kaucic, G. Mali, N. Novak Tusar and N. Zabukovec Logar acknowledge the financial support of the Slovenian Research Agency through the research program (P10021). VK, JRH and MH are grateful to The Royal Society, London and to the Slovenian Academy of Sciences and Arts for their financial and organisational support for numerous visits of VK to the University of Manchester and several visits of both JRH and MH to the National Institute of Chemistry, Ljubljana. We are grateful to the authors for their prompt handling of our permissions requests to reproduce their figures and/or commenting on the relevant text extracts as appropriate. Finally, owing to a bereavement in JRH's close family, this Keynote Lecture, prepared by the authors of this article, was kindly presented at the REXS 2011 Conference by Prof Jean-Louis Hodeau, to whom we are very grateful.

\section{References}

1. R. Kahn, R. Fourme, R. Bosshard, M. Chiadmi, J.L. Risler, O. Dideberg, J.P. Wery, FEBS Lett. 179, 133 (1985)

2. Y. Umena, K. Kawakami, J.-R. Shen, N. Kamiya, Nature 473, 55 (2011)

3. J. Jakoncic, M. Di Michiel, Z. Zhong, V. Honkimaki, Y. Jouanneau, V. Stojanoff, J. Appl. Cryst. 39, 831 (2006)

4. Y. Zhang, A.P. Wilkinson, P.L. Lee, S.D. Shastri, D. Shu, D.-Y. Chung, M.G. Kanatzidis, J. Appl. Cryst. 38, 433 (2005)

5. K. Djinovic Carugo, J.R. Helliwell, H. Stuhrmann, M.S. Weiss, J. Synchrotron Rad. 12, $410(2005)$

6. S. Ramaseshan, S.C. Abrahams (eds.) Anomalous Scattering, Munksgaard, Copenhagen (1975)

7. Resonant Anomalous X-ray Scattering; Theory and Applications, edited by G. Materlik, C.J. Sparks, K. Fischer, (North Holland, Amsterdam, 1994), pp. 675

8. A. Cheetham, A.P. Wilkinson, Angew. Chem. Int. Ed. Engl. 31, 1557 (1992)

9. J.L. Hodeau, V. Favre-Nicolin, S. Bos, H. Renevier, E. Lorenzo, J.-F. Berar, Chem. Rev. 101, 1843 (2001)

10. H. Flack, G. Bernardinelli, Acta Cryst. A55, 908 (1999)

11. A. Cassetta, A.M. Deacon, S.E. Ealick, J.R. Helliwell, A.W. Thompson J. Synchrotron Rad. 6, 822 (1999)

12. M. Cianci, J.R. Helliwell, M. Helliwell, V. Kaucic, N.Z. Logar, G. Mali, N.N. Tusar, Crystallography Reviews 11, 245 (2005)

13. J.B. Kortright, Resonant soft X-ray scattering from nanostructured magnetic materials these proceedings

14. D. Haskel, E. Kravtsov, Y. Choi, J.C. Lang, Z. Islam, G. Srajer, J.S. Jiang, S.D. Bader, P.C. Canfield, Eur. Phys. J. Special Topics 208, 141 (2012)

15. V. Favre-Nicolin, M.G. Proietti, C Leclere, N.A. Katcho, M.I. Richard, H. Renevier MAD, DAFS: a unique tool for studying Semiconductor Nanostructures, these proceedings

16. U. Shmueli, H.D. Flack, Acta Cryst. A66, 669 (2010)

17. N.M.D. Brown, J.B. McMonagle, G.N. Greaves J. Chem. Soc., Faraday Trans. I 80, 589 (1984) 
18. D.H. Templeton, L.T. Templeton, Acta Cryst. A41, 365 (1985)

19. M. Schiltz, G. Bricogne, Acta Cryst. D66, 447 (2010)

20. G. Wu, Y. Zhang, L. Ribaud, P. Coppens, C. Wilson, B.B. Iversen, F.K. Larsen, Inorg. Chem. 37, 6078 (1998)

21. J.M. Thomas, R. Raja, G. Sankar, R.G. Bell, D.W. Lewis, Pure Appl. Chem. 73, 1087 (2001)

22. M. Helliwell, J.R. Helliwell, V. Kaucic, N. Zabukovec Logar, S.J. Teat, J.E. Warren, E.J. Dodson, Acta Cryst. B66, 345 (2010)

23. Collaborative Computational Project, Number 4 Acta Cryst, D50, 760 (1994)

24. V. Petricek, M. Dusek, L. Palatinus, JANA2006. Institute of Physics, Praha, Czech Republic (2006)

25. G.M. Sheldrick, Acta Cryst. A64, 112 (2008)

26. M. Helliwell, R.H. Jones, V. Kaucic, N.Z. Logar, J. Synchrotron Rad. 12, 420 (2005)

27. Barber, J. Chem. Soc. Rev. 38, 185 (2009)

28. J. Yano, J. Kern, K.D. Irrgang, M.J. Latimer, U. Bergmann, P. Glatzel, Y. Pushkar, J. Biesiadka, B. Loll, K. Sauer, J. Messinger, A. Zouni, V.K. Yachandra, Proc. Natl. Acad. Sci. U.S.A. 102, 12047 (2005)

29. K.N. Ferreira, T.M. Iverson, K. Maghlaoui, J. Barber, S. Iwata, Science 19 March 2004: vol. 303, No. 5665, 1831

30. D.W.J. Cruickshank, Acta Cryst. D55, 583 (1999)

31. D.M. Blow, Acta Cryst. D58, 792 (2002)

32. H. Einspahr, K. Suguna, F.L. Suddath, G. Ellis, J.R. Helliwell, M.Z. Papiz Acta. Cryst. B41, 336 (1985)

33. A. Deacon, T. Gleichmann, A.J. Kalb (Gilboa), H. Price, J. Raftery, G. Bradbrook, J. Yariv, J.R. Helliwell, Faraday Transactions 93, 4305 (1997)

34. P.F. Henry, M.T. Weller, C.C. Wilson, J. Appl. Cryst. 36, 1361 (2003)

35. A. Wagner, et al., Diamond Light Source

36. H. Renevier, S. Grenier, S. Arnaud, J.F. Berar, B. Caillot, J.L. Hodeau, A. Letoublon, M.G. Proietti, B. Ravel, J. Synchrotron Rad. 10, 435 (2003)

37. M. Rice, S. Wakatsuki, A. Bienenstock, J. Appl. Cryst. 24, 598 (1991)

38. R. Serimaa, V. Eteläniemi, O. Serimaa, T. Laitalainen, A. Bienenstock, J. Appl. Cryst. 29, 390 (1996)

39. J.P. Hill, C.-C. Kao, D.F. McMorrow, Phys. Rev. B 55, R8662-R8665 (1997)

40. J.-P. Simon, D. Babonneau, M. Drouet, O. Lyon, J. Appl. Cryst. 42, 312 (2009)

41. M. Sztucki, E. Di Cola, T. Narayanan, J. Appl. Cryst. 43, 1479 (2010)

42. M. Sztucki, E. Di Cola, T. Narayanan, Eur. Phys. J. Special Topics 208, 319 (2012)

43. J.R. Helliwell, M. Helliwell, R.H. Jones, Acta Cryst. A61, 568 (2005)

44. M. Helliwell, B. Gallois, B. Kariuki, V. Kaucic, J.R. Helliwell, Acta Cryst. B49, 420 (1993)

45. J.R. Helliwell, J. Cryst. Growth 90, 259 (1988)

46. N.N. Tusar, S. Jank, R. Glaser, Chem. Cat. Chem. 3, 254 (2011)

47. S. Hau-Reige (2008) http://hasylab.desy.de/e70/e220/e33108/e35338/e35341/ infoboxContent35349/hauriege_eng.pdf 